\title{
RESEARCH ARTICLE Study on Emergency Monitoring and Deformation Law of Slope
}

\author{
Weizhao Chuan Zhuojia Chen \\ Guizhou Geology and Mineral Exploration and Development Bureau 115 Geological Brigade, Guiyang, Guizhou, \\ 551400 , China
}

\begin{abstract}
The slope of a project in Guiyang, due to heavy rain and other causes of serious deformation, the arrangement of 40 monitoring points, the slope of the emergency monitoring monitoring shows that the use of rapid and then accurate emergency method is feasible; Backfill pressure can stabilize the slope quickly. The supporting slope is characterized by strong deformation integrity and retrograde sliding. Guizhou is a mountainous area with many engineering slopes, which has reference value for emergency monitoring of similar slopes.
\end{abstract}

Keywords: Emergency monitoring; Emergency measures; Deformation law

Citation: Weizhao Chuan, Zhuojia Chen, 2018. Study on Emergency Monitoring and Deformation Law of Slope. International Journal of Geology, 3(1): 1-4. http://doi.org/10.26789/IJG.2018.01.001

Copyright: Study on Emergency Monitoring and Deformation Law of Slope. (C) 2018 Weizhao Chuan, Zhuojia Chen. This is an Open Access article published by Urban Development Scientific Publishing Company. It is distributed under the terms of the Creative Commons Attribution-Noncommercial 4.0 International License, permitting all non-commercial use, distribution, and reproduction in any medium, provided the original work is properly cited and acknowledged.

\section{Introduction}

With the economic and social development and the increase in the rate of urbanization, real estate projects have also made considerable progress and development, and the demand for underground garages in real estate projects is increasing. Therefore, during the excavation of the basement, a lot of zero-time or Permanent slope. This paper takes the zero-time slope in the basement excavation of a project in Guiyang as an example to study the slope deformation emergency monitoring method, precision control, slope stability measures, and slope deformation law. Due to continuous rainstorms, supporting conditions, groundwater and other factors, the slope has undergone severe deformation and subsidence. Wide cracks have appeared on the top of the slope, which endangers the safety of the construction operation of the foundation pit under the slope and the safety of the top of the slope. The roads are safe.

\section{Slope Emergency Monitoring Method and Precision Control}

\subsection{Monitoring Point Layout and Monitoring Frequency}

The length of the entire slope is about 280 meters, the height is $12-15$ meters, and the slope is 50-70 degrees. A total of 40 monitoring points are arranged in two rows at the upper and middle positions of the slope (as shown in Figure 1). Due to the cross construction, the bottom of the slope does not have the conditions for deployment. Because the slope is severely deformed, cracks are obvious, and the situation is urgent, it belongs to emergency rescue monitoring. The conventional deformation monitoring cycle can no longer meet the needs of the site, so a higher frequency monitoring method is adopted. The initial monitoring frequency is set at three times a day; after the slope stabilization measures are adopted, the amount of deformation is reduced, the frequency is adjusted to one to three days for monitoring, and finally to one to two weeks for monitoring.

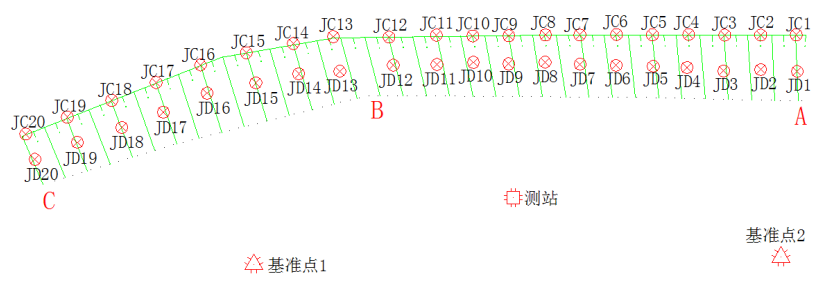

Figure 1. Schematic diagram of layout

\subsection{Monitoring Methods and Precision Control}

The Leica TS60 high-precision measurement system 
was used for this monitoring. Since each point can be seen through, the three-dimensional coordinates of each monitoring point are directly measured after the reference point of the rear view. The advantages of this method are simplicity, convenience, and high efficiency, but the disadvantage is that the accuracy of the measurement is not high enough. Due to the tight time and heavy tasks, in order to realize the slope monitoring earlier and faster, the monitoring method of quick first and accurate later was adopted. In the initial stage of the project, the monitoring method was adjusted in three stages to gradually improve the monitoring accuracy.

In the first stage, monitoring nails are buried at both the reference point and the monitoring point. The top of the monitoring nail is a cross fork, and a compulsory centering rod is erected on the top of the nail for measurement. The instrument is set up with a tripod. After measurement and comparison, the plane accuracy of this method is $3-6 \mathrm{~mm}$, and the elevation accuracy is $2-5 \mathrm{~mm}$, and the accuracy of single-center pole and single prism is higher than that of multiple-center pole and polygonal prism.

In the second stage, with the completion of the concrete embedded prism connector, the reference point and monitoring point are directly inserted into the pre-embedded prism connector for measurement, and the instrument is still set up with a tripod. After measurement and comparison, the plane accuracy of this method is $1-3 \mathrm{~mm}$, the elevation accuracy is $1-3 \mathrm{~mm}$, and the accuracy of a single prism is higher than that of a polygonal prism.

In the third stage, after the observation pier is buried and solidified and stabilized, the instrument is set up on the observation pier for measurement, and the reference point and monitoring point are directly inserted into the pre-embedded prism connector for measurement. After measurement and comparison, the plane accuracy of this method is $0.1-1 \mathrm{~mm}$, the elevation accuracy is $0.1-1 \mathrm{~mm}$, and the accuracy of a single prism is higher than that of a polygonal prism. This method can meet the requirements of high-precision monitoring.

\section{Study on the Deformation Law of the Slope of the Support Section}

According to the collected data and site surveys, the $\mathrm{AB}$ section of the slope is a supported slope. The supporting method is anchor rope and bolt, and the surface of the slope is sprayed with net. The BC section of the slope is an unsupported slope, which is mainly backfilled with earthy materials. The project has a total of 40 monitoring points, the observation time is about 100 days, and the number of observation periods is 50 periods. The article shows the data of 10 representative points and 10 periods.
The displacement data table in the horizontal direction is shown in Table 1, the displacement data table in the vertical direction is shown in Table 2, the horizontal displacement trend chart is shown in Figure 2, and the vertical displacement trend chart is shown in Figure 2.

Table 1. Data table of horizontal displacement (mm)

\begin{tabular}{ccccccccccc}
\hline Point & Day & Day & Day & Day & Day & Day & Day & Day & Day & Day \\
number & 1 & 3 & 5 & 10 & 15 & 20 & 30 & 50 & 80 & 100 \\
JC3 & 0.0 & 2.0 & 2.7 & 3.7 & 4.1 & 4.5 & 4.9 & 4.8 & 4.6 & 4.6 \\
JC5 & 0.0 & 3.4 & 4.8 & 8.4 & 12.1 & 13.9 & 13.8 & 14.3 & 14.9 & 15.1 \\
JC7 & 0.0 & 4.7 & 6.1 & 18.8 & 23.7 & 25.4 & 26.2 & 27.6 & 27.2 & 27.4 \\
JB7 & 0.0 & 8.6 & 12.3 & 25.4 & 33.7 & 35.9 & 37.2 & 39.6 & 38.8 & 39.7 \\
JC8 & 0.0 & 7.2 & 9.6 & 16.4 & 19.6 & 23.8 & 24.3 & 23.3 & 24.9 & 24.8 \\
JB8 & 0.0 & 9.1 & 14.0 & 23.9 & 28.7 & 30.9 & 33.7 & 35.5 & 36.9 & 36.4 \\
JC9 & 0.0 & 5.6 & 7.0 & 15.2 & 18.5 & 19.5 & 20.1 & 20.1 & 21.9 & 21.1 \\
JC10 & 0.0 & 4.9 & 6.3 & 7.4 & 8.3 & 11.8 & 11.3 & 12.1 & 11.0 & 10.2 \\
JC15 & 0.0 & 5.0 & 5.3 & 6.1 & 6.8 & 10.1 & 9.6 & 10.3 & 11.7 & 11.9 \\
JB15 & 0.0 & 4.2 & 5.4 & 6.3 & 6.9 & 8.2 & 9.8 & 10.3 & 9.9 & 8.7 \\
\hline
\end{tabular}

Table 2. Vertical displacement data table ( $\mathrm{mm})$

\begin{tabular}{ccccccccccc}
\hline Point & Day & Day & Day & Day & Day & Day & Day & Day & Day & Day \\
number & 1 & 3 & 5 & 10 & 15 & 20 & 30 & 50 & 80 & 100 \\
JC3 & 0.0 & -1.5 & -1.9 & -1.6 & -1.3 & 0.6 & -3.3 & -3.6 & -3.2 & -2.5 \\
JC5 & 0.0 & -6.8 & -11.9 & -16.1 & -18.1 & -20.0 & -21.5 & -22.4 & -22.3 & -22.6 \\
JC7 & 0.0 & -13.9 & -22.5 & -30.5 & -33.6 & -35.3 & -40.0 & -42.2 & -44.4 & -44.5 \\
JB7 & 0.0 & -10.5 & -13.2 & -22.5 & -26.2 & -28.3 & -29.0 & -30.1 & -30.3 & -30.8 \\
JC8 & 0.0 & -10.9 & -19.3 & -27.0 & -29.2 & -33.8 & -35.3 & -36.4 & -36.6 & -36.3 \\
JB8 & 0.0 & -8.9 & -10.7 & -16.6 & -22.8 & -23.4 & -23.4 & -24.5 & -25.2 & -25.9 \\
JC9 & 0.0 & -6.8 & -11.9 & -16.1 & -18.1 & -20.0 & -21.5 & -22.4 & -22.3 & -22.6 \\
JC10 & 0.0 & -5.1 & -6.2 & -6.9 & -8.0 & -10.3 & -10.7 & -11.8 & -12.4 & -13.1 \\
JC15 & 0.0 & -3.2 & -8.5 & -13.0 & -12.8 & -13.8 & -14.5 & -15.3 & -15.6 & -15.4 \\
JB15 & 0.0 & -4.1 & -4.6 & -13.6 & -14.4 & -13.0 & -16.4 & -14.7 & -15.3 & -15.9 \\
\hline
\end{tabular}

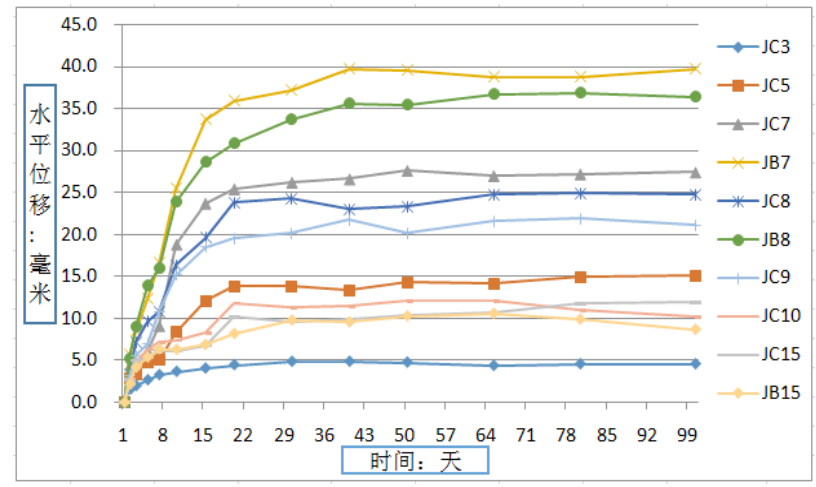

Figure 2. Trend chart of horizontal displacement 


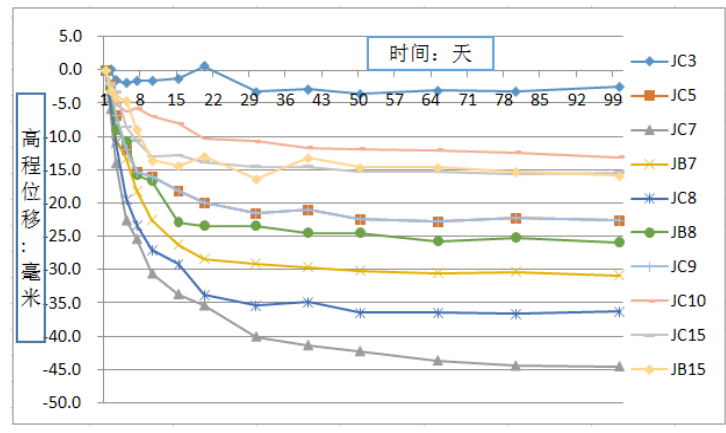

Figure 3. Vertical displacement trend graph

After the slope is deformed, in order to ensure the safety of people and property on the upper and lower sides of the slope and prevent further deformation of the slope, starting from the 5th day of monitoring, the slope is stabilized by backfilling with earth and rock under the slope until backfilling on the 20th day The work is basically completed. The backfilled earth and stone are distributed in strips, along the bottom of the slope, about 8 meters wide and 6-8 meters high. From Table 1, Table 2 and Figure 2 and Figure 3, the deformation law combined with the analysis of the construction situation, we get:

(1) According to the influence of backfilling pressure on the slope, the slope deformation can be divided into three stages:

The first stage is before backfilling. The time is $1-5$ days. The deformation rate is relatively large. The average displacement is $1.5 \mathrm{~mm}$ /day in the horizontal direction, 2.2 $\mathrm{mm}$ /day in the vertical direction, and the maximum point displacement is $2.8 \mathrm{~mm} /$ day in the horizontal direction. The sky, vertical direction is $4.4 \mathrm{~mm} /$ day;

The second stage is backfilling. The time is 5-20 days. The deformation rate gradually decreases. The average displacement is $0.8 \mathrm{~mm} /$ day in the horizontal direction, 0.6 $\mathrm{mm}$ /day in the vertical direction, and the maximum point displacement is $1.4 \mathrm{~mm}$ in the horizontal direction. /Day, $0.7 \mathrm{~mm} /$ day in the vertical direction;

The third stage is after backfilling, the time is $20-100$ days, the deformation rate is small, and the slope gradually stabilizes.

(2) The slope as a whole is deformed in the middle section as a whole, and gradually becomes smaller toward both sides. According to the analysis of monitoring data, the deformation in the JC1-JC5 direction gradually becomes larger; the JC5-JC9 section has the most serious deformation, with a cumulative maximum of $39.7 \mathrm{~mm}$ in the horizontal direction and $44.5 \mathrm{~mm}$ in the vertical direction; and a gradually smaller deformation in the direction of JC9-JC20 . Combining the deformation of other points, it is concluded that the displacement in the horizontal direction is proportional to the displacement in the vertical direction.

(3) The AB section is a supported slope. The top point of the slope (number JC) is about $12 \mathrm{~mm}$ smaller than the horizontal displacement of the middle point of the slope (number JB), and the top point of the slope is vertical The straight displacement is about $14 \mathrm{~mm}$. It can be concluded that the deformation of the slope in the supporting section becomes a "backward" sliding type sinking. The upper sinking is large and the translation is small, and the lower sinking is small and the translation is large. The integrity of the movement is strong. The BC section is an unsupported slope, and its deformation law is relatively scattered, with large randomness and weak integrity.

\section{Conclusions}

(1) Emergency rescue slope monitoring has the characteristics of tight time and heavy tasks. The monitoring frequency is much higher than the conventional monitoring frequency; the monitoring method requires fast, simple, and high-precision. In the case that all conditions cannot be met at the same time, it can be realized in stages according to different methods. The purpose of quickly understanding and grasping in time, and then precise control.

(2) In emergency slope rescue, it is feasible to use earth and rock backfilling to stabilize the slope, which has the characteristics of high efficiency, easy operation and good effect.

(3) The deformation of the slope has the characteristics of non-uniformity, segmentation, fragmentation, etc. The deformation law of the supported slope is different from that of the unsupported slope. The deformation of the supported slope is stronger overall, and it also has the characteristics of "backward" sliding type subsidence; while the deformation of the unsupported slope is not strong, fragmented, and random.

\section{References}

[1] Long Lin, Li Zhida. Research on monitoring and deformation law of a deep foundation pit project in Changsha City [J]. Building Structure, 2020, 50(02): 133-137.

[2] Gu Leiyu, Huang Hongwei, Chen Wei, et al. Early warning standards for safety risk of foundation pit construction in complex environments $[\mathrm{J}]$. Chinese Journal of Rock Mechanics and Engineering, 2020, 50(02):133-137.

[3] Pu Jianhua. Application research of highway slope monitoring and information management system[D]. Chengdu University of Technology, 2014. 
[4] Sun Huafen. Research on monitoring and prediction of Jianshan Phosphate Mine slope[D]. Kunming University of Science and Technology, 2014.

[5] Li Jinge. Research on Slope Monitoring System Based on Leica Survey Robot[D]. Chongqing Ji- aotong University, 2017.

[6] Li Xiufeng. Research on the surface risk monitoring and deformation law of subway foundation pit[D]. Anhui University of Science and Technology, 2018. 